\title{
Plantas de tratamiento de aguas residuales: estimación del consumo de drogas ilícitas en la zona oeste, Rio de Janeiro
}

\author{
Estações de tratamento de esgoto: estimativa do consumo de drogas ilícitas na \\ zona oeste, Rio de Janeiro
}

Wastewater treatment plants: assessment of illicit drug consumption in west zone, Rio de Janeiro

Aldo Pacheco Ferreira ${ }^{1 *}$, Maria José Cruz-Hernández².

\section{RESUMEN}

Objetivo: Estimar el consumo de drogas ilícitas [anfetamina (AMP), metanfetamina (METH), 3,4metilendioximetanfetamina (MDMA), cannabis (THC-COOH) y cocaína (COC)]. Métodos: Muestras compuestas (24 horas) de efluentes de 3 plantas de tratamiento de aguas residuales (PTAR) (Deodoro, Santa Cruz, Sepetiba), ubicadas en la zona oeste, Río de Janeiro, Brasil, fueron recolectadas en 2019 y posteriormente analizadas con la técnica de aguas residuales- epidemiología basada (WBE). Resultados: Las concentraciones de COC y sus metabolitos BE, Nor-BE y CE variaron de 36 a $601 \mathrm{ng} / \mathrm{L} ; 100$ a $889 \mathrm{ng} / \mathrm{L}$; $19,9 \mathrm{ng} / \mathrm{L}$ y $4,68 \mathrm{ng} / \mathrm{L}$, respectivamente. Los estimulantes similares a las anfetaminas variaron de $105 \mathrm{ng} / \mathrm{L}$ para AMP, $28 \mathrm{ng} / \mathrm{L}$ a $1674 \mathrm{ng} / \mathrm{L}$ para METH y 4,21ng/L para MDMA. THC-COOH osciló entre 112 y $178 \mathrm{ng} / \mathrm{L}$ y OHTHC 3,7ng/L. En relación con datos de consumo medio de drogas ilícitas entre 15 y 64 años (mg/día/1000 $\mathrm{Hab})$ solamente AMP fue detectada en la PTAR Deodoro (41,8 mg/día/1000 Hab), en cuanto a cannabis (marihuana) y cocaína, todas las PTAR fueron positivas. Conclusión: Se observaron diferencias significativas en la detección y consumo de las drogas estudiadas y se resaltó que el uso de drogas en el área estudiada no es homogéneo, pero suscita preocupación con los posibles impactos en la salud pública.

Palabras clave: Drogas ilícitas, Epidemiología basada en aguas residuales, Salud pública, Violencia, Planta de tratamiento de aguas residuales.

\section{RESUMO}

Objetivo: Estimar o consumo de drogas ilícitas [anfetaminas (AMP), metanfetamina (METH), 3,4metilenodioximetanfetamina (MDMA), cannabis (THC-COOH) e cocaína (COC)]. Métodos: Amostras compostas (24 horas) de efluentes de 3 estações de tratamento de esgoto (ETE) (Deodoro, Santa Cruz, Sepetiba), localizadas na zona oeste, Rio de Janeiro, Brasil, foram coletadas em 2019 e, posteriormente analisadas com a técnica de epidemiologia baseada em águas residuais (WBE). Resultados: As concentrações de COC e seus metabólitos BE, Nor-BE e CE variaram de 36 a $601 \mathrm{ng} / \mathrm{L} ; 100$ a 889ng/L; $19,9 \mathrm{ng} / \mathrm{L}$ e $4,68 \mathrm{ng} / \mathrm{L}$, respectivamente. Estimulantes semelhantes às anfetaminas variaram de $105 \mathrm{ng} / \mathrm{L}$ para AMP, 28ng/L a 1674ng/L para METH e 4,21ng/L para MDMA. THC-COOH variou entre 112 e 178ng/L e o metabólito $\mathrm{OH}-\mathrm{THC}$ de 3,7ng/L. Em relação aos dados de consumo médio de drogas ilícitas entre 15 e 64 anos (mg/dia/1000 hab.), apenas foi detectado AMP na ETE Deodoro (41,8 mg/dia/1000 hab.), em termos de cannabis (maconha) e cocaína, todas as ETEs foram positivas. Conclusão: Se observou diferenças relevantes na detecção e consumo das drogas estudadas, ressaltando que o uso de drogas na área estudada não é homogêneo, porém suscita preocupação com os possíveis impactos à saúde pública.

Palavras-chave: Drogas ilícitas, Epidemiologia baseada em esgoto, Saúde pública, Violência, Estação de tratamento de esgoto.

\footnotetext{
${ }^{1}$ Escola Nacional de Saúde Pública Sergio Arouca (ENSP/FIOCRUZ), Rio de Janeiro - RJ.

*E-mail: aldoferreira@ensp.fiocruz.br

${ }^{2}$ Comisión Nacional contra las Adicciones (CONADIC), Ciudad de México, México.
}

Pesquisa financiada pelo CNPq

SUBMETIDO EM: 12/2021

ACEITO EM: 1/2022

PUBLICADO EM: 2/2022 


\begin{abstract}
Objective: To estimate the consumption of illicit drugs [amphetamine (AMP), methamphetamine (METH), 3,4methylenedioxymethamphetamine (MDMA), cannabis (THC-COOH) and cocaine (COC)]. Methods: Composite samples (24 hours) of effluents from 3 wastewater treatment plants (WWTP) (Deodoro, Santa Cruz, Sepetiba), located in the west zone, Rio de Janeiro, Brazil, were collected in 2019 and later analysed with the technique of wastewater-based epidemiology (WBE). Results: The concentrations of COC and its BE, Nor$\mathrm{BE}$ and CE metabolites, ranged from 36 to $601 \mathrm{ng} / \mathrm{L} ; 100$ to $889 \mathrm{ng} / \mathrm{L} ; 19.9 \mathrm{ng} / \mathrm{L}$ and $4.68 \mathrm{ng} / \mathrm{L}$, respectively. Amphetamine-like stimulants ranged from 105ng/L for AMP, 28ng/L to 1674ng/L for METH and $4.21 \mathrm{ng} / \mathrm{L}$ for MDMA. THC-COOH ranged from 112 to $178 \mathrm{ng} / \mathrm{L}$ and the $\mathrm{OH}-\mathrm{THC}$ metabolite of 3.7ng/L. Regarding data on average consumption of illicit drugs between 15 and 64 years (mg/day/1000 inhab), only AMP was detected at WWTP Deodoro (41.8 mg/day/1000 inhab), in terms of cannabis (marijuana) and cocaine, all WWTP were positive. Conclusion: Relevant differences were observed in the detection and consumption of the studied drugs, emphasizing that drug use in the studied area is not homogeneous, but raises concerns about the possible impacts on public health.
\end{abstract}

Keywords: Illicit drugs, Wastewater-based epidemiology, Public health, Violence, Wastewater treatment plant.

\title{
INTRODUCCIÓN
}

El uso de sustancias ilícitas ha sido un punto de interés mundial desde el inicio del comienzo de la civilización. La necesidad de mejorar la calidad de vida ha obligado a las autoridades a vigilar los hábitos sociales, incluido el consumo de sustancias ilícitas. La aspiración de supervisar tales sustancias surge de su potencial para causar graves problemas de salud, así como de sus posibles reacciones adversas (DEGENHARDT L, et al., 2018).

El uso indebido de drogas ilícitas es una preocupación mundial y se considera una carga grave para la salud pública y el bienestar socioeconómico. Por lo tanto, la lucha contra el problema de las drogas requiere medidas complejas que involucran a diferentes organismos gubernamentales (DEGENHARDT L, et al., 2018).

Cabe señalar que el uso de drogas ilícitas se ha coligado con conductas violentas por muchas décadas, bien como evidencia la prevalencia de enfermedades mentales concurrentes (KEEN C, et al., 2022). Si bien la relación es la misma hoy que en el pasado, la omnipresencia de la asociación y las consecuencias son más dramáticas. Hay dos formas en las que el abuso de sustancias está relacionado con la violencia. En primer lugar, la violencia puede perpetrarse y se perpetra bajo la influencia de sustancias y, en segundo lugar, la violencia relacionada con el abuso de sustancias se deriva del comercio de drogas, que con demasiada frecuencia se concentra en comunidades pobres y desatendidas (ASCHNER JP e MONTERO JC, 2021).

Para lograr estos objetivos, los responsables de la enunciación de políticas demandan datos racionales de consumo, realistas, basados en pruebas y en tiempo real. Por esta razón, en las últimas décadas se adoptó un enfoque de indicadores múltiples para una estimación más precisa del consumo de drogas a nivel comunitario. Este enfoque de indicadores múltiples incluye herramientas de apoyo adicionales como la epidemiología de alcantarilla (WBE) para colmar los vacíos (como sesgos y demoras) de otros métodos convencionales según la European Monitoring Centre for Drugs and Drug Addiction (EMCDDA) (EMCDDA, 2016).

La WBE utiliza la reunión de basuras de drogas ilícitas y metabolitos en la descarga de aguas residuales para monitorear la prevalencia del uso de tales sustancias en la sociedad. Para lograr esto, las drogas progenitoras medidas y/o sus metabolitos urinarios estables se someten a un paso de cálculo inverso, que requiere parámetros como el caudal diario, el factor de excreción y la población atendida por la planta de tratamiento de aguas residuales (PTAR) relacionada (ZUCCATO E, et al., 2005).

Por la eficiencia y objetividad de los resultados, la fácil accesibilidad y la rentabilidad de los ejemplares, se ha manejado como parte de la determinación de las tasas de consumo de sustancias ilícitas en la sociedad desde principios de la década de 2000 (EMCDDA, 2016). Esta idea fue introducida por primera vez por 
Daughton CG (2001), quien propuso un puente entre el medio ambiente y las ciencias sociales. En 2005, Zuccato desarrolló un procedimiento analítico novedoso manejando cocaína a manera sustancia modelo, que constituyó una base general para la epidemiología de las aguas residuales (ZUCCATO E, et al., 2005).

Durante las últimas décadas, varios científicos han perfeccionado métodos analíticos sofisticados para evaluar el consumo de drogas ilícitas con el apoyo del Observatorio Europeo de las Drogas y las Toxicomanías (EMCDDA) y el Sewage Analysis CORe group Europe (VAN NUIJS AL, et al., 2011; REID MJ, et al., 2012; THOMAS KV, et al., 2012; CASTIGLIONI S, et al., 2013; ORT C, et al., 2014).

WBE se ha aplicado con éxito para estimar hábitos que pueden dañar la salud pública (FERREIRA AP, 2019a). Mientras tanto, los campos de aplicación de la WBE han sido poco difundidos debido a su aspecto interdisciplinario; sin embargo, la mayoría de los estudios que utilizan la tecnología WBE en todo el mundo se han centrado en las drogas ilícitas (DENG Y, et al., 2020). Sin embargo, la vigilancia de las drogas ilícitas sigue siendo el campo más popular de la WBE debido al alcance de los problemas mundiales de las drogas y a la necesidad de datos basados en pruebas para que los responsables de formular políticas establezcan políticas objetivas (GAO T, et al., 2017).

Como la United Nations Office on Drugs and Crime (UNODC) ha mencionado muchas veces en World Drug Reports, Brasil está expuesto a la ruta del narcotráfico de varios países productores de América del Sur hacia Europa y Estados Unidos (UNODC, 2018). Luego, debido a la posición geográfica de Brasil, es crucial monitorear las tendencias cambiantes de las drogas para comprender mejor los patrones de consumo de drogas a corto y largo plazo, especialmente en ciudades cosmopolitas, sobre la base de mediciones de aguas residuales de drogas de interés (FRAGA PCP, 2007; FERREIRA AP, 2019b).

El municipio de Rio de Janeiro es la capital del estado de Rio de Janeiro, ubicada en la Región Sudeste, Brasil. Es el centro del turismo y el entretenimiento en todas las estaciones y tiene una gran diversidad de personas, vida empresarial y lugares históricos. Por lo tanto, se cree que las tendencias de drogas y los datos de consumo de la ciudad pueden ser útiles para crear una perspectiva de todo el país. Por lo tanto, investigar las tendencias del consumo de drogas ilícitas in extenso del tiempo logra ofrecer un panorama representativo del país debido al tamaño y al carácter cosmopolita de la población. Por lo tanto, el estudio presentado fue delineado para investigar la importancia de un monitoreo completo casi en tiempo real de las tendencias temporales de las drogas en Rio de Janeiro en 2020/2021 (SOUSA E, et al., 2013).

El objetivo fue estimar el consumo de drogas ilícitas convencionales [anfetamina (AMP), metanfetamina (METH), 3,4-metilendioximetanfetamina (MDMA), cannabis y cocaína] a partir de las concentraciones obtenidas de influente debido consumo en el sitio de estudio por moradores con edad de 15 a 64 años. Además, se evaluaron las variaciones estacionales entre las 3 PTAR ubicadas en la zona oeste. Así con los resultados se puede ayudar en la implementación de medidas basadas en evidencia.

\section{MÉTODOS}

\section{Descripción de la zona de muestreo}

Río de Janeiro es un municipio brasileño perteneciente al Estado de Rio de Janeiro y representa el segundo mayor Producto Interno Bruto (PIB) del país. El municipio de Rio de Janeiro posee como área total $1224,56 \mathrm{~km}^{2}$. Se encuentra situado a 230' $10^{\prime \prime} \mathrm{S}$ y $43^{\circ} \div 7^{\prime} 40^{\prime \prime} \mathrm{W}$ (INSTITUTO PEREIRA PASSOS, 2021). La población considerada por el Instituto Brasileño de Geografía y Estadística (IBGE) en 2021 es de 6.775.561 habitantes (IBGE, 2021).

El presente trabajo investigó las PTAR ubicadas en la zona oeste de Río de Janeiro, más específicamente en el Área de Planificación 5 (AP5). Actualmente, los servicios de alcantarillado sanitario en la AP5 están a cargo de Concesionaria Foz Aguas, subsidiario de la Compañía Estadual de Agua e Esgotos (CEDAE), y que opera en la región desde 2012, a través de un Contrato de Concesión con el Municipio de Río de Janeiro. La Concesionaria opera diecinueve PTAR en la AP5, sin embargo, doce de ellas son PTAR pequeñas, cuyo caudal no supera los $10 \mathrm{~L} / \mathrm{s}$. Así, las PTAR Deodoro, PTAR Santa Cruz y PTAR Sepetiba seleccionadas para el estudio son las que presentan características relevantes, especialmente en cuanto a caudal y ubicación (CEDAE, 2021). 
La PTAR Deodoro opera los efluentes de los barrios de Bangú, Deodoro, Realengo, Padre Miguel, Magalhães Bastos, Jardim Sulacap y Vila Militar. Posee capacidad para atender a 430.000 personas, es la estación más grande dentro de la concesión de saneamiento en la AP5, que corresponde al $48 \%$ del territorio municipal. Cuenta con dos módulos con una capacidad total para tratar $800 \mathrm{~L} / \mathrm{s}$ de aguas residuales, lo que representa el tratamiento del $100 \%$ del $97 \%$ de las aguas residuales recolectadas en la ciudad. Cada módulo está compuesto por laguna aireada, laguna de sedimentación y laguna de lodo (CEDAE, 2021).

La PTAR Santa Cruz trata un promedio de $450 \mathrm{~m}^{3}$ por día de aguas residuales y cuenta con un sistema de tratamiento tipo fangos activados con $90 \%$ de rendimiento en el tratamiento de efluentes. La carga orgánica promedio del afluente es de $855 \mathrm{mg} / \mathrm{L}$ y el efluente $91 \mathrm{mg} / \mathrm{L}$. Tiene su operación de recolección de aguas residuales en los barrios de Santa Cruz, Paciência, Cosmos, Inhoaíba y Campo Grande (CEDAE, 2021).

En la PTAR Sepetiba La captación de aguas servidas llega al 100\% de los hogares y las aguas residuales recolectadas son tratadas en su totalidad, aunque el sistema de tratamiento es bastante rudimentario, restringido a estanques anaeróbicos y de estabilización facultativa, sin impermeabilizaciones ni ningún control aparente. la carga orgánica promedio del afluente es de $683 \mathrm{mg} / \mathrm{L}$ y el efluente de $85 \mathrm{mg} / \mathrm{L}$ (CEDAE, 2021).

\section{Recogida de muestras}

Las pruebas de control y prolijidad del muestreo, conservación y exámenes son esenciales para aseverar la entereza de la espécimen comenzando en su cosecha hasta el reportaje de los resultados; contiene la prontitud de seguir o monitorear las circunstancias de toma de ejemplar, conservación, categorización, envío y su ulterior análisis. Este proceso es elemental y significativo para puntear atención y reserva de la muestra (BIJLSMA L, et al., 2018).

Las muestras del influente se recolectaron aplicando un muestreador automático a través de técnica de muestreo-composta en abril/2021, por un período de 24h, según las orientaciones establecidas por Ort C, et al. (2010) y Castiglioni S, et al. (2013). Todas las muestras de aguas residuales sin tratar se recogieron en contenedores de polietileno de alta densidad de $3 \mathrm{~L}$ mediante el uso de un muestreador automático programado para muestrear $200 \mathrm{ml}$ cada $60 \mathrm{~min}$.

\section{Compuestos analizados}

Con el fin de investigar la presencia de drogas ilícitas y sus metabolitos en plantas de tratamiento de aguas residuales de la zona Oeste de Río de Janeiro, se efectuaron análisis con las concernientes muestras, utilizando la metodología de extracción de fase sólida, cromatografía líquida y espectrometría de masas (HPLC-MS/MS) basado en Senta I, et al. (2013).

Los análisis de las muestras fueran hechas en el Laboratorio de análisis químico (espectrometría de masas) de la Universidad Jaume I (UJI), Castellón, Espanã, pesquisando drogas y metabolitos de clases distintas de compuestos químicos análogos a las anfetaminas, cannabinoides y cocaínicos, tales como: Cocaína (COC), Benzoilecgonina (BE), Nor-Benzoilecgonina (Nor-BE), Cocaetileno (CE), Anfetamina (AMP), Metanfetamina (METH), 3,5-metilenedioximetamfetamina (MDMA o éxtasis), $\Delta$-tetrahidrocannabinol (THC), 11-nor-9-carboxi- $\Delta$-tetrahidrocannabinol (THC-COOH), 11-hidroxi- $\Delta$-tetrahidrocannabiol (OH-THC).

Los procedimientos analíticos aplicados para la determinación de drogas ilícitas y sus metabolitos (Tabla 1) en las aguas residuales han cambiado poco desde los resultados de Thomas KV (2012), salvo algunas modificaciones y mejoras derivadas de una mejor comprensión del destino de los biomarcadores en el alcantarillado (CAUSANILLES A, et al., 2017), onde se manejaron técnicas analíticas validadas, que generalmente consistieron en: (i) agregar muestras con estándares internos marcados con isótopos estables para cada analito, con el fin de perfeccionar las interferencias de la matriz o mermas durante el tratamiento de la muestra; (ii) filtración o centrifugación de muestras para eliminar partículas sólidas y (iii) extracción en fase sólida (SPE).

La elaboración de cada análisis consistió en su dilución 1/4, extracción en fase sólida (cartuchos Oasis $\mathrm{HLB}$ ), vaporización a $35^{\circ} \mathrm{C}$ en nitrógeno y su restablecimiento en $1 \mathrm{~mL}$ de metanol al $10 \%$. El examen cromatográfico fue ejecutado con la utilización de cromatógrafo de líquidos de ultra alta resolución Waters 
Acquity UHPLC (Comalcalco, Tabasco, México) tangente a un espectrómetro de masas de triple cuadrupolo (Xevo TQS, Waters Micromass, Ciudad de México, México). El volumen de muestra introducido fue de $20 \mu l$. La concentración de cada compuesto se enunció en ng/L de muestra de agua residual.

Tabla 1 - Drogas ilícitas y metabolitos analizados, abreviaturas, fórmulas, números CAS, límites de detección y factores de corrección. Rio de Janeiro, 2021.

\begin{tabular}{|c|c|c|c|c|c|}
\hline Grupos de drogas & Drogas y metabolitos & Abreviaturas & Números $\mathrm{CAS}^{*}$ & $\begin{array}{l}\text { Límites de } \\
\text { detección } \\
\text { (ng/L) }\end{array}$ & $\begin{array}{l}\text { Factor de } \\
\text { corrección }\end{array}$ \\
\hline \multirow{3}{*}{ Anfetaminas } & Anfetamina & AMP & $300-62-9$ & 1,28 & - \\
\hline & Metanfetamina & METH & $537-46-2$ & 0,25 & - \\
\hline & 3,5-metilendoximetanfetamina & MDMA & $42542-10-9$ & 0,38 & 1,513 \\
\hline \multirow{3}{*}{ Cannabinoides } & $\Delta^{9}$-tetrahidrocannabinol & THC & $1972-08-3$ & 5 & 2,04 \\
\hline & $\begin{array}{l}11 \text {-hidroxi- } \Delta^{9} \text { - } \\
\text { tetrahidrocannabiol }\end{array}$ & $\mathrm{OH}-\mathrm{THC}$ & $36557-05-8$ & 2,5 & 0,37 \\
\hline & $\begin{array}{l}11 \text {-nor-9-carboxi- } \Delta^{9} \text { - } \\
\text { tetrahidrocannabinol }\end{array}$ & $\mathrm{THC}-\mathrm{COOH}$ & $23978-85-0$ & 60 & 1,26 \\
\hline \multirow{4}{*}{ Cocaínicos } & Cocaína & $\mathrm{COC}$ & $50-36-2$ & 2,5 & - \\
\hline & Benzoilecgonina & $\mathrm{BE}$ & $519-09-5$ & 10 & 2,3313 \\
\hline & Nor-Benzoilecgonina & Nor-BE & $(-)$ & 2,5 & 3,71 \\
\hline & Cocaetileno & CE & $529-38-4$ & 5 & 4,781 \\
\hline
\end{tabular}

Subtitular: *Los números CAS son identificadores numéricos asignados a cada compuesto químico puntualizado en la literatura académica. Esta concesión ejecuta la Sociedad Americana de Química, Chemical Abstracts Service (CAS). (-): No existe número CAS. -: No aplica.

Fuente: Ferreira AP y Cruz-Hernández MJ, 2022.

\section{Estimación del consumo a través del análisis de aguas residuales}

Para obtener la cantidad de consumo de drogas investigada en el sitio de estudio, es expresar, $\mathrm{mg} / \mathrm{d}$ áa/1.000Hab, se obtuvo el cómputo de la cantidad detectada de cada compuesto, y este contenido se dividió por la cantidad de población atendida por las respectivas PTARs. Para evaluar el consumo de drogas a partir de la concentración hallada en influente, se midieron las sustancias parentales (anfetamina, metanfetamina y MDMA) y dos metabolitos urinarios (benzoilecgonina para la cocaína y THC-COOH para el cannabis), con base en el procedimiento propuesto por Daughton CG (2001) y utilizado por Zuccato E, et al. (2008).

Se procedió, por lo tanto, a formular la cantidad estimada de drogas consumidas con los cálculos establecidos por Zuccato E, et al. (2008). Así, la cantidad de fármaco consumido por día (ng/día) se logró multiplicando la concentración del metabolito (ng/L) por el caudal de agua que ingresó a la PTAR el día del muestreo (L/día). El contenido de cada metabolito se ajustó con un factor de corrección, conforme puntualizado en la Tabla 1, según Nefau T, et al. (2013).

\section{Análisis estadístico}

El análisis de los datos para obtener la concentración de las drogas y sus respectivos metabolitos se expresa mediante el consumo promedio de cada producto y la desviación estándar. Vale la pena señalar que las estimaciones de la concentración de los metabolitos detectados, se correspondieron con la ubicación de la PTAR. Todos los análisis se realizaron con el programa estadístico SPSS versión 24.

\section{Consideraciones éticas}

El presente trabajo contó con la asentimiento del Comité de Ética en Investigación Científica de la Escola Nacional de Saúde Pública Sergio Arouca (Ensp/Fiocruz), bajo el Dictamen 07/2018.

\section{RESULTADOS}

Se encontraron metabolitos de drogas en contenidos cuantificables, tales como: THC-COOH, cocaína, benzoilecgonina, anfetamina y metanfetamina. Además, el consumo de cannabis, cocaína, anfetamina y metanfetamina a nivel poblacional se estimó en agua residual de cada PTAR. 
En la Tabla 2 es presentado la concentración mediana de drogas y metabolitos cuantificados, obtenidos en influentes de las PTARs, de acuerdo con su ubicación. Anfetamina fue cuantificada en PTAR Deodoro (153 ng/L) y PTAR Sepetiba (105 ng/L) y detección en la PTAR Santa Cruz exhibió límite inferior de cuantificación. La mayoría de las PTAR tuvieron niveles cuantificables de metanfetamina, con la concentración más alta en PTAR Deodoro (1674 ng/L). Los niveles cuantificados más altos de THC-COOH se midieron en la PTAR Sepetiba (178 ng/L). Los niveles más altos de cocaína se encontraron en PTAR Deodoro (601 ng/L), mismamente a manera la concentración más alta de benzoilecgonina (889 ng/L).

Tabla 2 - Media de la concentración de drogas ilícitas y metabolitos (ng/L) en las muestras recolectadas (PTAR Deodoro, PTAR Santa Cruz y PTAR Sepetiba). Rio de Janeiro, 2021.

\begin{tabular}{ccccc}
\hline Grupos de drogas & Drogas y metabolitos & $\begin{array}{c}\text { PTAR } \\
\text { Deodoro }\end{array}$ & $\begin{array}{c}\text { PTAR Santa } \\
\text { Cruz }\end{array}$ & PTAR Sepetiba \\
\hline \multirow{3}{*}{ Anfetaminas } & AMP & 153 & $<\mathrm{LQ}$ & 105 \\
& $\mathrm{METH}$ & 1674 & 28 & 388 \\
& $\mathrm{MDMA}$ & 4,21 & $<\mathrm{LQ}$ & $<\mathrm{LQ}$ \\
\hline \multirow{3}{*}{ Cannabinoides } & $\mathrm{THC}$ & $<\mathrm{LQ}$ & $<\mathrm{LQ}$ & $<\mathrm{LQ}$ \\
& $\mathrm{OH}-\mathrm{THC}$ & 3,7 & $<\mathrm{LQ}$ & $<\mathrm{LQ}$ \\
& $\mathrm{THC}-\mathrm{COOH}$ & 178 & 112 & 136 \\
\hline \multirow{3}{*}{ Cocaínicos } & $\mathrm{COC}$ & 601 & 36 & 118 \\
& $\mathrm{BE}$ & 889 & 100 & 552 \\
& $\mathrm{Nor}-\mathrm{BE}$ & 19,9 & $<\mathrm{LQ}$ & $<\mathrm{LQ}$ \\
& $\mathrm{CE}$ & 4,68 & $<\mathrm{LQ}$ & $<\mathrm{LQ}$ \\
\hline
\end{tabular}

Subtitular: <LQ: límite inferior de cuantificación; anfetamina (AMP); metanfetamina (METH); 3,5metilenedioximetamfetamina (MDMA (éxtasis)); $\Delta$-tetrahidrocannabinol (THC); 11-nor-9-carboxi- $\Delta$ tetrahidrocannabinol (THC-COOH); 11-hidroxi- $\Delta$-tetrahidrocannabiol (OH-THC); Cocaína (COC); Benzoilecgonina (BE); Nor-benzoilecgonina (Nor-BE); Cocaetileno (CE).

Fuente: Ferreira AP y Cruz-Hernández MJ (2022).

En la Tabla 3 se muestran los niveles de consumo de anfetamina, metanfetamina, marihuana y cocaína, de acuerdo con la ubicación de la PTAR. Anfetamina fue cuantificada únicamente en la PTAR Deodoro (41,8 $\mathrm{mg} / \mathrm{día} / 1000 \mathrm{Hab}$ ), en cuanto a marihuana y cocaína, en todas las PTAR estuvieron positivas. La metanfetamina se cuantificó en todas PTAR; la ubicada en Deodoro reportó el consumo más alto (50 $\mathrm{mg} / \mathrm{día} / 1000 \mathrm{Hab}$ ). Igualmente, los niveles cuantificados de cocaína y marihuana también mostraron mayor consumo en la población relacionada con la PTAR Deodoro $(345 \mathrm{mg} / \mathrm{día} / 1000 \mathrm{Hab}$ y $620 \mathrm{mg} / \mathrm{día} / 1000 \mathrm{Hab}$, respectivamente).

Tabla 3 - Consumo medio ( \pm desviación estándar) de drogas ilícitas entre 15 y 64 años (mg/día/1000 Hab) en las muestras recolectadas (PTAR Deodoro, PTAR Santa Cruz y PTAR Sepetiba). Rio de Janeiro, 2021.

\begin{tabular}{ccccc}
\hline \multicolumn{2}{c}{ Grupo de drogas } & PTAR Deodoro & PTAR Santa Cruz & PTAR Sepetiba \\
\hline \multirow{3}{*}{ Anfetaminas } & AMP & $40,10( \pm 5,22)$ & no detectado & no detectado \\
& METH & $50( \pm 17,50)$ & $12( \pm 1,6)$ & $11( \pm 2,30)$ \\
& MDMA & $120( \pm 16,99)$ & no detectado & no detectado \\
\hline Cannabis (THC) & & $345( \pm 47,84)$ & $182( \pm 25,34)$ & $111( \pm 23,11)$ \\
\hline Cocaína (COC) & $620( \pm 112,09)$ & $46( \pm 11,27)$ & $38( \pm 8,22)$ \\
\hline
\end{tabular}

Fuente: Ferreira AP y Cruz-Hernández MJ, 2022.

\section{DISCUSIÓN}

La WBE es una disciplina científica en rápido desarrollo con el potencial de monitorear datos en tiempo real sobre tendencias geográficas y temporales en el uso de drogas ilícitas, que se ha establecido como un complemento útil a las herramientas de monitoreo existentes en el campo de las drogas ilícitas. Implica tomar muestras de una fuente de aguas residuales, como las aguas residuales afluentes de una planta de tratamiento de aguas residuales. Esto permite a los científicos medir los niveles de drogas ilícitas y sus metabolitos excretados, que luego pueden usarse para estimar la cantidad de drogas consumidas por una comunidad. Esto proporciona un análisis no invasivo y casi en tiempo real del uso de sustancias en la región utilizada por la red de alcantarillado muestreada. 
Se detectaron además los metabolitos Nor-BE y cocaetileno (que son metabolitos de la cocaína) en la PTAR Deodoro, exhibiendo concentraciones más elevadas que en otros estudios realizados (NEFAU T, et al., 2013; GAO T, et al., 2017; SULEJ-SUCHOMSKA AM, et al., 2020).

La detección del metabolito THC-COOH exhibe la segunda concentración más alta en influente de las PTARs analizadas. La concentración detectada es similar a la encontrada por Bijlsma L, et al. (2016), que igualmente detectó THC-COOH y OH-THC. La presencia de esta sustancia en las muestras analizadas se debe a que a pesar de solamente $20 \%$ del THC consumido sea excretado por la orina.

Respecto al grupo de las anfetaminas, se obtuvo la presencia de metanfetamina (METH) en todas PTAR analizadas; la anfetamina (AMP) se detectó en dos PTAR (Deodoro y Sepetiba); y el éxtasis (MDMA) solamente en la PTAR Deodoro.

El consumo medio de AMP en ciudades como Barcelona (MASTROIANNI N, et al., 2017), Berlín (RYU Y, et al., 2016), Helsinki (LÖVE ASC, et al., 2018), Oslo (BAZ-LOMBA JA, et al., 2016; LÖVE ASC, et al., 2018), Estocolmo (LÖVE ASC, et al., 2018) y Nueva York (SUBEDI B y KANNAN K, 2014) estuvo con niveles mayores a los datos obtenidos (PTAR Deodoro - 40,1 mg/día/1000Hab). Sin embargo, al comparar la región estudiada con las ciudades utilizadas como parámetro, es proporcionalmente un consumo elevado.

En otro orden de ideas, fue observado que el consumo de METH (PTAR Deodoro - $50 \mathrm{mg} / \mathrm{día} / 1000 \mathrm{Hab}$ ), (PTAR Santa Cruz - $12 \mathrm{mg} / \mathrm{d}$ áa/1000Hab) y (PTAR Sepetiba - $11 \mathrm{mg} / \mathrm{día} / 1000 \mathrm{Hab})$ se presentó en menor escala como en otras ciudades, por ejemplo, Nueva Zelanda (KUMAR R, et al., 2019), Sureste de Queensland (LAI FY, et al., 2016), Barcelona (MASTROIANNI N, et al., 2017) y también en megaciudades chinas (KHAN $\mathrm{U}$, et al., 2014), que mostraron consumo análogo entre ellos (120 mg/día/1000Hab).

Cuando se comparó la cantidad de consumo de MDMA, se comprobó que la PTAR Deodoro poseía un consumo equivalente o superior $(120 \mathrm{mg} / \mathrm{día} / 1000 \mathrm{Hab})$ al de otras ciudades como Barcelona (MASTROIANNI N, et al., 2017), Utrecht (BAZ-LOMBA JA, et al., 2016) y Queensland (LAI FY, et al., 2016). No obstante, se encontró que la cocaína era el estimulante más consumido en PTAR Deodoro (620 mg/día/1000Hab), se observó que el consumo estaba muy por encima del promedio en comparación con el de otras ciudades como Helsinki (LÖVE ASC, et al., 2018), Zagreb (KRIZMAN-MATASIC I, et al., 2019), y megaciudades chinas (KHAN U, et al., 2014). PTAR Santa Cruz y PTAR Sepetiba estuvieron entre los resultados comparados con las grandes ciudades que mostraron la menor cantidad de consumo de cocaína. Se encontró que la marihuana, que fue la droga ilícita más consumida en la mayoría de los estudios, se consumía en cantidades más bajas (PTAR Deodoro - $345 \mathrm{mg} / \mathrm{día} / 1000 \mathrm{Hab}$ ), (PTAR Santa Cruz - $182 \mathrm{mg} / \mathrm{día} / 1000 \mathrm{Hab}$ ) y (PTAR Sepetiba - $111 \mathrm{mg} / \mathrm{día} / 1000 \mathrm{Hab}$ ) en comparación, por ejemplo, con Barcelona (3607 mg/día/100 Hab) (MASTROIANNI N, et al., 2017).

La información precisa y oportuna sobre la escala y la dinámica del consumo de drogas es importante para evaluar las necesidades de los servicios policiales y de salud pública en una comunidad. El análisis cuidadoso de las muestras de aguas residuales puede proporcionar información valiosa sobre la escala del consumo de drogas, pero con una investigación exhaustiva de la cinética del flujo de sustancias ilícitas, tal procedimiento técnico también puede proporcionar un apoyo valioso para identificar tendencias en los patrones de consumo de drogas. Dicha información puede agregar un peso cuantitativo adicional a los hallazgos de las encuestas sociológicas y de población en general.

\section{CONCLUSIÓN}

Comprender el consumo de drogas ilícitas sigue siendo una tarea difícil; sin embargo, los dados obtenidos de esta averiguación enseñan cómo la combinación de fuentes de identificaciones diferentes y complementarias permiten obtener una imagen más precisa de la situación. La información se puede utilizar para monitorear los cambios en el uso de drogas, identificar peligros potenciales, promover la configuración de encuestas específicas, campañas de prevención y/o acciones policiales), comprender la estructura de los compradores de sustancias ilícitas y situar las políticas antidrogas futuras. Aunque el análisis de aguas residuales no proporciona información directa sobre los usuarios, su capacidad para proporcionar datos casi en tiempo real y su viable combinación en los programas de monitoreo existentes lo convierten en una herramienta valiosa para ayudar a comprender el consumo de drogas ilícitas. 


\section{FINANCIAMENTO}

El estudio fue financiado por el Conselho Nacional de Desenvolvimento Científico e Tecnológico (CNPq), proceso 301966/2017-6.

\section{AGRADECIMIENTOS}

Agradecer el apoyo de la Comisión Nacional Contra las Adicciones-Conadic (Centro Nacional para la Prevención y el Control de las Adicciones-Cenadic) y CEDAE (Companhia Estadual de Água e Esgotos).

\section{REFERENCIAS}

1. ASCHNER JP, MONTERO JC. Architectures, spaces, and territories of illicit drug trafficking in Colombia and Mexico. Crime, Media, Culture, 2021; 17(3):327-351.

2. BAZ-LOMBA JA, et al. Comparison of pharmaceutical, illicit drug, alcohol, nicotine and caffeine levels in wastewater with sale, seizure and consumption data for 8 European cities. BMC Public Health, 2016; 16: 1-11.

3. BIJLSMA L, et al. Análisis de aguas residuales con fines epidemiológicos: aplicaciones a la estimación del consumo de sustancias de abuso y en salud pública en general. Revista Española de Salud Pública, 2018; 92: e201808053.

4. BIJLSMA L, et al. Estimation of illicit drug use in the main cities of Colombia by means of urban wastewater analysis. Science of the Total Environment, 2016; 565: 984-993.

5. CASTIGLIONI S, et al. Evaluation of uncertainties associated with the determination of community drug use through the measurement of sewage drug biomarkers. Environmental Science \& Technology, 2013; 47(3): 1452-1460.

6. CAUSANILLES A, et al. Occurrence and fate of illicit drugs and pharmaceuticals in wastewater from two wastewater treatment plants in Costa Rica. Science of the Total Environment, 2017; 599-600: 98-107.

7. CEDAE. Companhia Estadual de Água e Esgotos. Disponível em: http://www.cedae.com.br/. Acessado em: 12 de maio de 2021.

8. COSENZA A, et al. Occurrence of illicit drugs in two wastewater treatment plants in the South of Italy. Chemosphere, 2018; 198: 377e385.

9. DAUGHTON CG. Illicit Drugs in Municipal Sewage. Pharmaceuticals and Care Products in the Environment. Washington, DC: American Chemical Society, 2001.

10. DEGENHARDT $L$, et al. The associations between psychotic experiences, and substance use and substance use disorders: findings from the World Health Organization World Mental Health surveys. Addiction, 2018; 113: 924-934.

11. DENG Y, et al. Occurrence and removal of illicit drugs in different wastewater treatment plants with different treatment techniques. Environmental Sciences Europe, 2020; 32: 28.

12. EUROPEAN MONITORING CENTRE FOR DRUGS AND DRUG ADDICTION (EMCDDA). Assessing illicit drugs in wastewater: advances in wastewater-based drug epidemiology, Insights 22. 2016. Publications Office of the European Union, Luxembourg. Disponível em: https://doi.org/10.2810/017397. Acessado em: 12 de maio de 2021.

13. FERREIRA AP. Illicit Drugs in Wastewater Treatment Plants A case study: Rio de Janeiro, Brazil. Journal of Chemical Health Risks, 2019; 9(3): 191-202.a

14. FERREIRA AP. Estimaciones del consumo de drogas ilícitas derivadas del análisis de aguas residuales: Una revisión crítica. Revista de la Universidad Industrial de Santander. Salud, 2019; 51(1); 69-80.b

15. FERREIRA AP, CRUZ-HERNÁNDEZ MJ. Evaluación del consumo de drogas ilícitas per cápita por epidemiología de alcantarilla: impacto en el medio ambiente y salud pública. Revista Eletrônica Acervo Saúde, 2021; 13(3): 1-10.

16. FRAGA PCP. A geopolítica das drogas na América Latina. Revista da Faculdade de Serviço Social da Universidade do Estado do Rio de Janeiro, 2007; (19): 67-88.

17. GAO T, et al. Occurrence of new psychoactive substances in wastewater of major Chinese cities. Sci Total Environ. 2017; 575: 963-969.

18. IBGE. Instituto Brasileiro de Geografia e Estatística. Disponível em: https://www.ibge.gov.br/cidades-e-estados/rj/riode-janeiro.html. Acessado em: 17 de dezembro de 2021.

19. INSTITUTO PEREIRA PASSOS. Áreas de Planejamento do Rio de Janeiro. 2021. Disponível em: http://www.data.rio/datasets/31d845e1c56a49c6a2b0e01420ec83b8. Acessado em: 12 de maio de 2021.

20. KEEN C, et al. A retrospective cohort study using linked health, income assistance, corrections and death records. Setting British Columbia (BC), Canada. Addiction, 2022; 117(1): 129-140.

21. KHAN U, et al. Application of a sewage-based approach to assess the use of ten illicit drugs in four Chinese megacities. Science of the Total Environment, 2014; 487: 710-721.

22. KRIZMAN-MATASIC I, et al. Long-term monitoring of drug consumption patterns in a large-sized European city using wastewater-based epidemiology: Comparison of two sampling schemes for the assessment of multiannual trends. Science of the Total Environment, 2019; 647: 474-485. 
23. KUMAR R, et al. Assessment of drugs of abuse in a wastewater treatment plant with parallel secondary wastewater treatment train. Science of the Total Environment, 2019; 658: 947-957.

24. LAI FY, et al. Cocaine, MDMA and methamphetamine residues in wastewater: Consumption trends (2009-2015) in South East Queensland, Australia. Science of the Total Environment, 2016; 568: 803-809.

25. LÖVE ASC, et al. Analysis of stimulant drugs in the wastewater of five Nordic capitals. Science of the Total Environment, 2018; 627: 1039-1047.

26. MASTROIANNI N, et al. Five-year monitoring of 19 illicit and legal substances of abuse at the inlet of a wastewater treatment plant in Barcelona (NE Spain) and estimation of drug consumption patterns and trends. Science of the Total Environment, 2017; 609: 916-926.

27. NEFAU T, et al. Presence of illicit drugs and metabolites in influents and effluents of 25 sewage water treatment plants and map of drug consumption in France. Science of the Total Environment, 2013; 461-462: 712-722.

28. ORT C, et al. Sampling for pharmaceuticals and personal care products (PPCPS) and illicit drugs in wastewater systems: are your conclusions valid? A Critical Review. Environmental Science \& Technology, 2010; 44: 6024-6035.

29. REID MJ, et al. Estimation of cocaine consumption in the community: a critical comparison of the results from three complimentary techniques. BMJ Open, 2012; 2(6): e001637.

30. RYU Y, et al. Comparative measurement and quantitative risk assessment of alcohol consumption through wastewater-based epidemiology: An international study in 20 cities. Science of the Total Environment, 2016; 565: 977-983.

31. SENTA I, et al. Integrated procedure for multiresidue analysis of dissolved and particulate drugs in municipal wastewater by liquid chromatography-tandem mass spectrometry. Analytical and Bioanalytical Chemistry, 2013; 405(10): 3255-3268.

32. SOUZA, ER, et al. Consumo de substâncias lícitas e ilícitas por policiais da cidade do Rio de Janeiro. Ciência \& Saúde Coletiva, 2013; 18(3): 667-676.

33. SUBEDI B, KANNAN K. Mass loading and removal of select illicit drugs in two wastewater treatment plants in New York State and estimation of illicit drug usage in communities through wastewater analysis. Environmental Science \& Technology, 2014; 48: 6661-6670.

34. SULEJ-SUCHOMSKA AM, et al. Urban wastewater analysis as an effective tool for monitoring illegal drugs, including new psychoactive substances, in the Eastern European region. Sci Rep. 2020; 10: 4885.

35. THOMAS KV, et al. Comparing illicit drug use in 19 European cities through sewage analysis. Science of the Total Environment, 2012; 432: 432-9.

36. UNODC. Global overview of drug demand and supply. 2018. Disponível em: https://www.unilibrary.org/content/books/9789210450584c003. Acessado em: 12 de maio de 2021.

37. VAN NUIJS AL, et al. Illicit drug consumption estimations derived from wastewater analysis: A critical review. Science of the Total Environment, 2011; 409(19): 3564-3577.

38. ZUCCATO E, et al. Identification of the pharmaceuticals for human use contaminating the Italian aquatic environment. Journal of Hazardous Materials, 2005; 122: 205-209. 